\title{
Anti-osteoporotic Effects of Unripe Fructus of Rubus coreanus Miquel in Osteoblastic and Osteoclastic Cells
}

\author{
Hyo Jin Kim, Dong-Soo Sim and Eun-Hwa Sohn* \\ Department of Herbal Medicine Resource, Kangwon National University, Samcheok 245-907, Korea
}

\begin{abstract}
Osteoporosis is a progressive bone disease characterized by low bone mass which is caused by disturbance in the balance between the activities of osteoblasts and osteoclasts. Postmenopausal osteoporosis is one of the most common disorders in women after menopause, which is linked to an estrogen deficiency and characterized by an excessive loss of trabecular bone. Rubus coreanus has been used for their various pharmacological properties in Asia as a traditional medicine. To investigate the effect of unripe fruits of $R$. coreanus $30 \%$ ethanol extract (RCE) on osteoblast-like cells (MG63) differentiation, we examined the effects of RCE on in vitro osteoblastic differentiation markers, alkaline phosphatase (ALP) activity and receptor activator of nuclear factor $\mathrm{K}-\mathrm{B}$ ligand (RANKL) and osteoprotegerin (OPG) expression. The high concentration (50 and 100 $\mu \mathrm{g} / \mathrm{mL}$ ) of RCE markedly increased ALP activity, whereas decreased the RANKL/OPG. We also investigated the effect of RCE on M-CSF plus RANKL-induced differentiation of pre-osteoclast cells (RAW 264.7). RCE treatment remarkably inhibited M-CSF/RANKL-induced formation of osteoclast-like multinuclear cells from RAW 264.7 cells. Moreover, the inhibitory effect of RCE was reduced by selective estrogen receptor- $\alpha$ antagonist. Our research suggests that suggested that unripe fruits of $R$. coreanus may act beneficial effects on bone mass by regulating both osteoblast and osteoclast.
\end{abstract}

Key words - Osteoblast, Osteoclast, Osteoporosis, RANKL, Rubus coreanus

\section{Introduction}

Bone is continuously broken-down and re-formed in a process of turnover known as bone remodeling through the interaction and balance between bone forming cells, called osteoblasts, and bone resorbing cells, called osteoclasts (Karsenty, 2003). A disturbance in the tight balance between the activities of osteoblasts and osteoclasts causes problems with bone mass such as excessive gain of bone mass (osteopetrosis) and loss of bone mass (osteoporosis). Osteoporosis is a progressive bone disease characterized by low bone mass and deterioration of bone tissue which occurs far more frequently and is a major burden on health-care systems globally (McNamara, 2010).

The osteoblasts produce, organize, and mineralize various bone matrix proteins including type I collagen, which is the most abundant extracellular bone protein (Aubin and Triffitt, 2002). The process of osteoblast differentiation and matrix mineralization requires a rise in high enzyme activity of

${ }^{*}$ Corresponding author. E-mail : ehson@kangwon.ac.kr alkaline phosphatase (ALP) and the responses to osteotropic hormones and cytokines. Moreover, in the process of bone formation, the osteoblasts regulate osteoclast differentiation and resorption activity via cell-cell contact, whereby RANKL (receptor activator of nuclear factor $\kappa \mathrm{B}$ ligand) binds to its cognate receptor, RANK (receptor activator of nuclear factor $\kappa-B)$. Osteoprotegerin (OPG) is one of the tumor necrosis factor receptor (TNFR) superfamily members which plays as a decoy receptor for RANKL to inhibit osteoclast differentiation and decrease bone resorption by competing with the binding of RANKL to RANK (Kostenuik and Shalhoub, 2001). RANKL and OPG synthesized by stromal osteoblasts have been identified as the two principal cytokines of osteoclast differentiation and activation (Kostenuik and Shalhoub, 2001; Hamdy, 2007). The binding of RANKL to RANK results in the activation of signaling pathways, regulating osteoclast function (Khosla, 2001; Udagawa, 2000). Thus, the OPG/ RANKL/RANK signal pathway is considered as a key cytokine system implicated in the control of bone remodeling.

Osteoporosis is most often considered a bone disorder of 
postmenopausal women which is linked to an estrogen deficiency. It has been well established that estrogen deficiency causes the overexpression of pro-inflammatory cytokines and increases osteoclast activity provoking bone resorption, which leads to a high bone turnover and bone loss as well (Kearns et al., 2008; Fraser et al., 2011; Wensel et al., 2011).

Since estrogen deficiency is known to cause bone loss by stimulating osteoclast formation, hormone replacement therapy (HRT) was used in the prevention and treatment of postmenopausal osteoporosis. However, several studies have suggested that long-term estrogen therapy such as $17 \beta$ estradiol may be associated with the risks of blood clots, biliary disease, breast, and endometrial cancers (Marsden, 2002). Therefore, plant-derived estrogenic compounds may be great benefit to postmenopausal women, as they have similar structures with estrogen and/or have the ability to bind to estrogen receptors (Miksicek, 1994). The estrogen-like activity of these compounds may provide an alternative strategy to replace or augment the HRT to prevent bone loss, avoiding the major side effects seen with HRT (Geller and Kronenberg, 2003).

$R$. coreanus (Rosaceae) is a native species of raspberry from Korea, Japan and China. The dried unripe fruits of $R$. coreanus are widely used as traditional medicines for treating spermatorrhea, and enuresis, and have also shown stomachic and tonic actions (Kim et al., 2005). The various biological effects of $R$. coreanus include anti-oxidative, anti-fatigue, increasing fertility, improvement of libido, astringent, antiosteoporotic, ophthalmic, anti-diabetic and estrogenic effects (Jung et al., 2007; Kim et al., 2013). It is well known that $R$. coreanus contains various bioactive compounds, including phenolic acids, triterpenosides, flavonoids, and ellagitannin (Jang et al., 2011). It was also reported that plant-derived polyphenol, ellagic acid, one of the bioactive compounds in the fruits of $R$. coreanus, was significantly higher in unripe fruits than ripe fruits of $R$. coreanus (Yang et al., 2008). Recently, it was found protocatechuic acid, a polyphenolic compound in unripe fruits of $R$. coreanus which elicits antioxidative and anti-inflammatory effects (Koo et al., 2014b; Lee et al., 2014). With regard to anti-osteoporotic effects of $R$. coreanus, there are several reports based on cell line studies, demonstrating that $R$. coreanus treatment increased the differentiation of osteoblastic cells, MC3T-ET1 (Park et al., 2006), and accelerated the apoptosis of pro-osteoclastic cells, RAW 264.7 (Lee and Choi, 2006). The anti-osteoporotic effects of $\mathrm{R}$. coreanus have been shown through studies in vivo, where it elicited protective effects against bone loss in diabetic osteoporosis induced by streptozotocin in ovariectomized rats (Choi et al., 2012), and recovery effects against trabecular bone loss in OVX-rats in the form of a methanol extract, which could not be clinically applied (Do et al., 2008). Based on these studies, we previously suggested the optimal ratio for the R. coreanus and A. membranaceus mixture to be $7: 3$ by weight, as this mixture resulted in the anti-osteoporotic effects in ovariectomy-induced mice (Koo et al., 2013; Koo et al., 2014a).

In the present study, we aimed to clarify the anti-osteoporotic effects of EtOH extracts of $R$. coreanus that could be used for clinical studies using both of the two type of cells, osteoblast and osteoclast. We determined the regulatory effects of RCE on osteoblastic differentiation and osteoclast differentiation under the same experimental conditions. Moreover, we sought to define regulatory factors involved in the anti-osteoporotic effects of $R$. coreanus including ALP activity in osteoblast and RANKL/OPG expression and the association of estrogenic receptor in osteoclast differentiation.

\section{Materials and Methods}

\section{Preparation of $\boldsymbol{R}$. coreanus unripe fruits extract (RCE)}

The dried unripe fruits $R$. coreanus was purchased in April, 2012 from Gyeong-dong Herbal Market (yak-ryong-si, Seoul, Korea). Voucher specimen (KNU-HMR-2013) was deposited in the department of Herbal Medicine Resource at Kangwon National University. Each of the dried fruits (1000 g) was extracted twice with ethanol $(30 \% \mathrm{EtOH})$ at room temperature and the extract was concentrated under reduced pressure. The concentrate was filtered, lyophilized, and subsequently stored at $4{ }^{\circ} \mathrm{C}$.

\section{Materials and reagents}

Unless otherwise indicated, all chemicals were purchased from Sigma Chemical Co. (St Louis, MO, USA). Dulbecco's modified Eagle's medium (DMEM), RPMI 1640 medium, 
penicillin, streptomycin, trypsin-EDTA and fetal bovine serum (FBS) were purchased from Life Technologies, Inc. (Carlsbad, CA, USA). The antibodies against RANKL, OPG, and $\beta$-actin were obtained from Santa Cruz Biotechnology (Santa Cruz, CA, USA).

\section{Cell culture and cell viability}

The human osteoblastic cell line, MG63 and the murine macrophage cell line, RAW 264.7 were purchased from the American Type Culture Collection (ATCC, Rockville, MD, USA). The MG63 cells were cultured in DMEM medium containing $10 \% \mathrm{FBS}$ and antibiotics $(100 \mathrm{IU} / \mathrm{ml}$ of penicillin and $100 \mu \mathrm{g} / \mathrm{ml}$ of streptomycin) and incubated at $37^{\circ} \mathrm{C}$ in a humidified atmosphere containing $5 \% \mathrm{CO}_{2}$. Osteoclast precursor cells, RAW 264.7 (ATCC, Rockville, MD, USA) was maintained in RPMI 1640 medium containing 10\% FBS. Cells were cultured at a concentration of $5 \times 10^{4}$ cells/well in 96-well culture plates and treated with RCE for $24 \mathrm{~h}$ and then 3-[4,5-dimethylthiazol-2-yl]-2,5-diphenyltetrazolium bromide (MTT; Sigma) solution was added into each well. The crystal violet generated by viable cells was dissolved with $10 \%$ sodium dodecyl sulfate (SDS; Sigma) solution and optical density (O.D.) was measured at $570 \mathrm{~nm}$ using a microplate reader (Molecular devices, Menlo Park, CA, USA).

\section{Analysis of alkaline phosphatase (ALP) activity}

MG63 cells $\left(2 \times 10^{4}\right)$ were seeded on 6-well plates and incubated with reagents for 4 days. The cell layer was then washed with D-PBS and dissolved in $0.25 \%$ TritonX-100. The cell lysate was then sonicated for $30 \mathrm{sec}$ on ice. The cellular protein concentration was determined after incubation in a protein assay reagent; bovine serum albumin (BSA) containing $0.25 \%$ TritonX-100 was used as a standard. The ALP activity was measured using a spectrophotometric method with para-nitrophenyl-phosphate as the substrate. The optical density (O.D.) was measured at $405 \mathrm{~nm}$ using a microplate reader. The results, which were normalized to protein concentration, are expressed as a percentage of the control.

\section{Westem blot analysis}

The expression of RANKL and OPG was determined by Western blot analysis. After 4 days incubation with or without
RCE, cells were harvested and lysed in ice-cold lysis buffer (50 mM Tris- $\mathrm{HCl}$ [pH 7.4], $150 \mathrm{mM} \mathrm{NaCl}, 1 \mathrm{mM}$ EDTA, $0.5 \%[\mathrm{v} / \mathrm{v}] \mathrm{NP}-40,0.1 \%[\mathrm{w} / \mathrm{v}] \mathrm{SDS})$ containing protease inhibitor cocktail for $1 \mathrm{~h}$. Twenty micrograms of each sample was electrophoresed on $10 \%$ SDS-PAGE and transferred to nitrocellulose membranes. The membranes were blocked with 5\% skim milk in Tris-buffered saline/Tween-20 for $1 \mathrm{~h}$ before being probed with the appropriate antibodies. They were then washed with Tris-buffered saline/Tween-20 once for $15 \mathrm{~min}$, and then 3 times for $5 \mathrm{~min}$, prior to being incubated with secondary HRP-conjugated antibody for $1 \mathrm{~h}$. The blots were developed using an enhanced chemiluminescence kit. In all immunoblotting experiments, the blots were reported with an antibody against $\beta$-actin, which was used as protein loading control.

\section{In vitro osteoclast differentiation}

The murine pre-osteoclast cell line RAW 264.7 cells were cultured in 24-well dishes at a density of $1 \times 10^{4}$ cells per well and allowed to adhere overnight. To induce osteoclastic differentiation, RAW 264.7 was cultured with $150 \mathrm{ng} / \mathrm{ml}$ RANKL and $100 \mathrm{ng} / \mathrm{ml}$ M-CSF. After 2 days in M-CSF/RANKL media, RCE was added to the cultures and after 4 days in culture, TRAP staining was performed. The number of TRAPpositive multinucleated osteoclasts was detected by microscopy.

\section{Statistical analysis}

Each experiment was repeated at least two times, and the results of one representative experiment are shown. The results were expressed as means \pm SEM. Group differences were determined by one-way analysis of variance (ANOVA), followed by modified t-test with the Bonferroni correction for comparisons between individual groups. The significant values are represented by an asterisk $\left({ }^{*}{ }^{*} p<0.05\right)$.

\section{Results and Discussion}

The present study provides evidence that dried unripe fruit of $R$. coreanus could be significantly effective in the prevention and treatment of osteoporosis with affecting on both of the osteoblastic and osteocalstic cells. The cytotoxic effects of RCE on MG63 and RAW 264.7 cells were assessed using the 

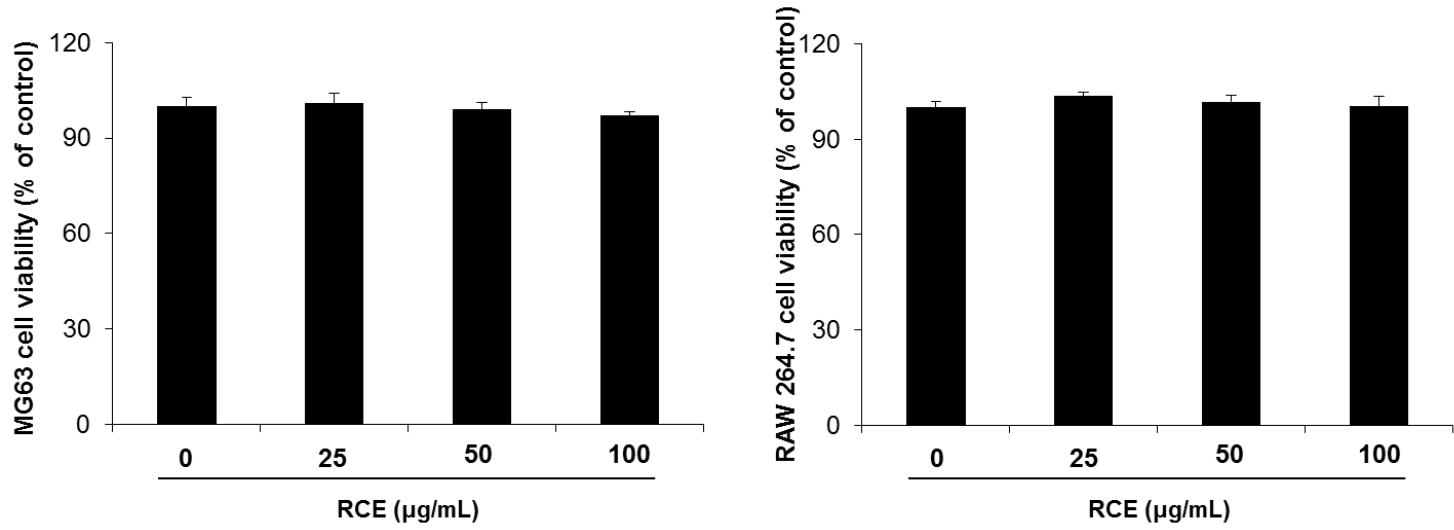

Fig. 1. Effect of RCE on cell viability in MG63 and RAW 264.7. Analysis of cell viability using the MTT assay following $24 \mathrm{~h}$ treatment with various concentrations of RCE. These results are means \pm S.E.M of quintuplicates from a representative experiment.

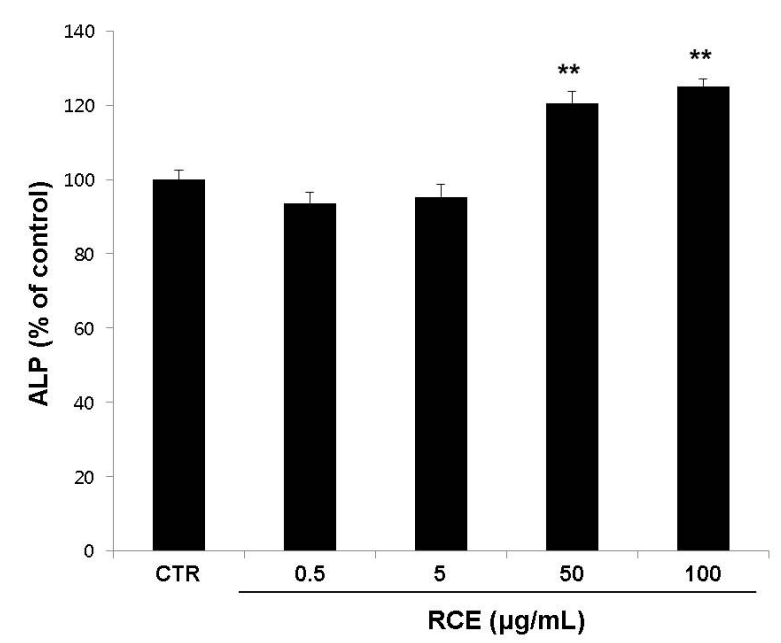

Fig. 2. Effects of RCE on the ALP activity in MG-63 cells. These results are means \pm S.E.M of quintuplicates from a representative experiment. ${ }^{* *} \mathrm{P}<0.01$ : significantly different from the untreated control group.

MTT assay. Results demonstrated that RCE did not affect cell viability and was not cytotoxic to both cells at the concentrations used (Fig. 1).

ALP activity is one of the major osteoblastic differentiation markers. The effect of RCE on osteoblastic differentiation was determined by the treatment of MG63 cells with $0.5,5$, 50 , and $100 \mu \mathrm{g} / \mathrm{ml}$ of RCE for 4 days. As shown in Fig. 2, ALP activity was significantly increased by the high concentration (50 and $100 \mu \mathrm{g} / \mathrm{ml}$ ) of RCE. However, low concentrations of RCE $(0.5$ and $5 \mu \mathrm{g} / \mathrm{ml})$ did not affect ALP activity. This suggests that RCE treatment alone can induce osteoblastic cell differentiation. Because the significant stimulatory effect of RCE on ALP activity was observed at high concentrations, in subsequent experiments, cells were treated with 25,50 , and $100 \mu \mathrm{g} / \mathrm{ml}$ of RCE.

In the present study, we reported that beneficial effect of RCE on the osteoblast differentiation. To elucidate this observation, we hypothesized that the RCE could regulate osteoblast secretion of crucial regulatory factors such as RANKL and OPG (Simonet et al., 1997; Thomas et al., 2001). Therefore, we determined protein levels of RANKL and OPG in MG63 cells treated with 25, 50, and $100 \mu \mathrm{g} / \mathrm{ml}$ RCE. Our results indicate that high concentrations of RCE treatment significant decreased the level of RANKL (Fig. 3), whereas there was no significant effect on the levels of OPG (Fig. 4). As a consequence, the RANKL/OPG ratio was decreased significantly by RCE treatment at higher doses (50 and 100 $\mu \mathrm{g} / \mathrm{ml})$. The differentiation of osteoclast cell precursors is mediated upon the binding of RANKL and RANK, which promotes the activation of mature osteoclasts. Therefore, RANKL is an essential factor for differentiation, activation, and survival of osteoclasts, whereas osteoprotegerin (OPG) is a soluble decoy receptor and inhibitor of RANKL (Aubin and Bonnelye, 2000). The OPG and RANKL are important regulators of osteoclastogenesis which are produced by osteoblastic cells and the RANKL/OPG ratio could be used to assess and manage patients with severe osteolysis (Horwood et al., 1998; Thomas et al., 2001). Our results suggest that RCE could inhibit the osteoclastogenesis by regulation of osteoblast function.

Next, we investigated the effect of RCE on M-CSF plus 

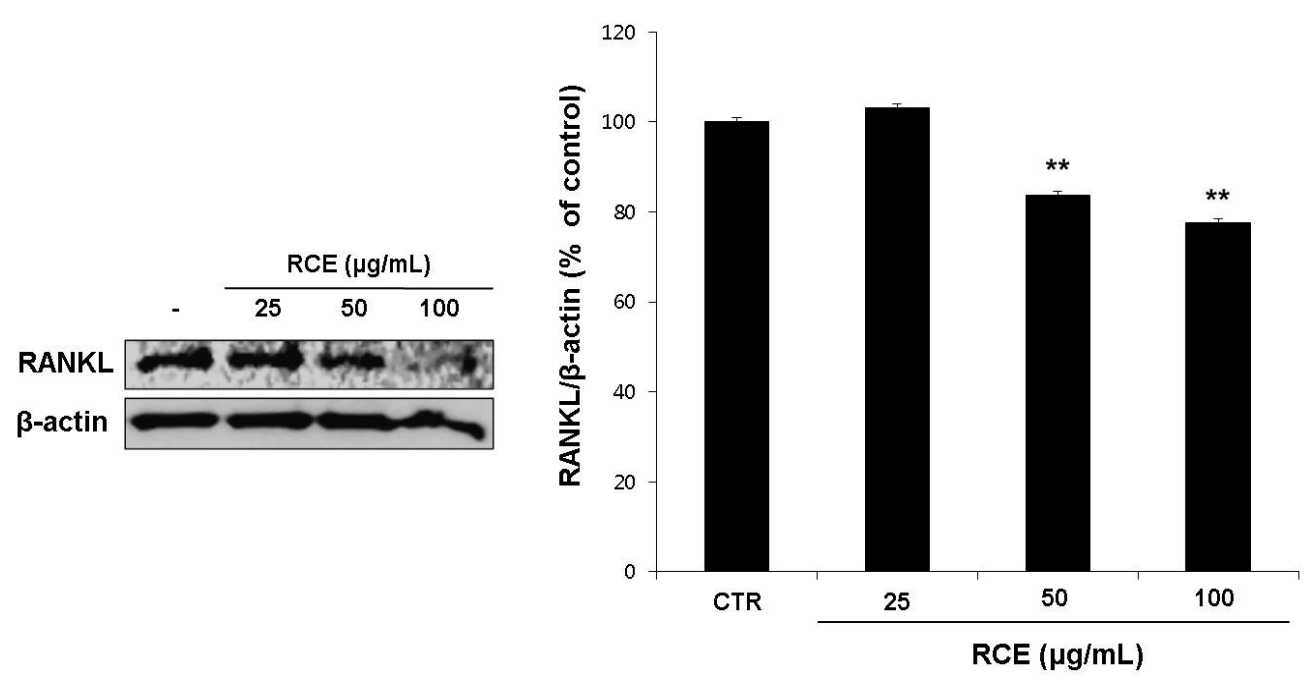

Fig. 3. Effects of RCE on the expression of RANKL in MG-63 cells. Cells were incubated with RCE (25, 50, and $100 \mu \mathrm{g} / \mathrm{ml})$ for 4 days and cell lysates were prepared and subjected to Western blot analysis with respective antibodies. These experiments were performed in triplicate, and similar results were obtained.
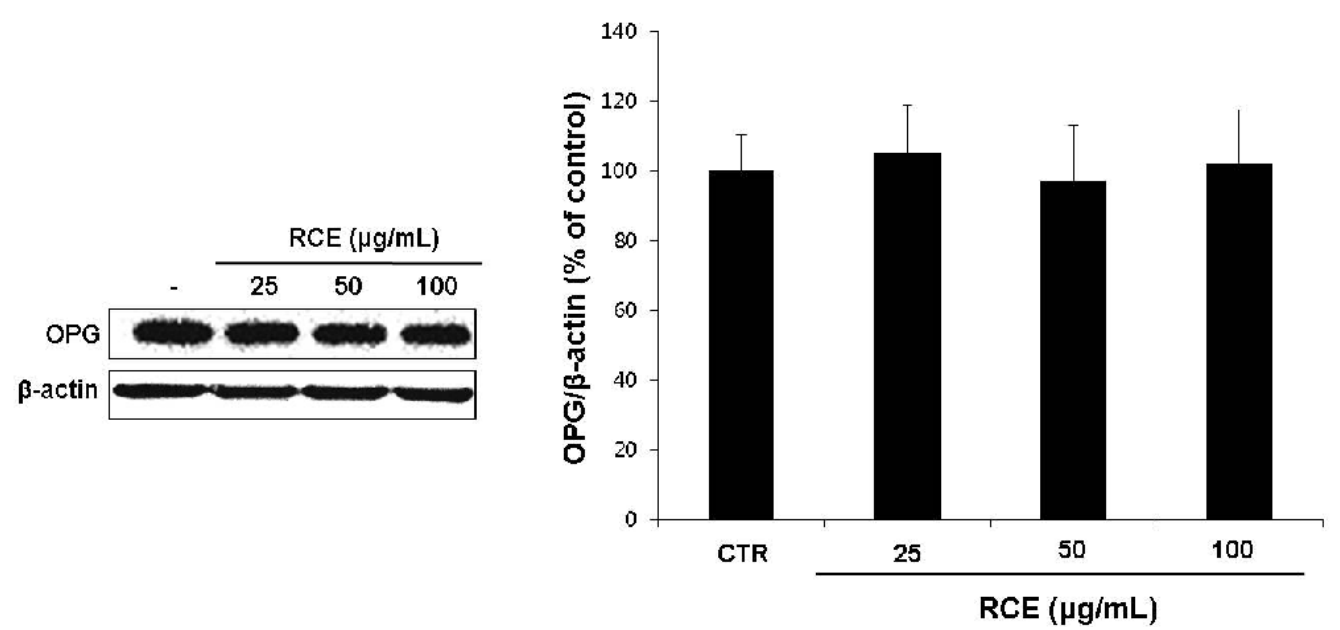

Fig. 4. Effects of RCE on the expression of OPG in MG-63 cells. Cells were incubated with RCE $(25,50$, and $100 \mu \mathrm{g} / \mathrm{ml})$ for 4 days and cell lysates were prepared and subjected to Western blot analysis with respective antibodies. These experiments were performed in triplicate, and similar results were obtained.

RANKL-induced osteoclastogenesis. RAW 264.7 cells were incubated with different concentrations of RCE in the presence of M-CSF/RANKL and allowed to differentiate into osteoclasts. Fig. 5 illustrates that M-CSF/RANKL induced osteoclasts at day 4. By contrast, RCE treatment remarkably inhibited M-CSF/RANKL-induced formation of osteoclast-like multinuclear cells from RAW 264.7 cells in a concentrationdependent manner (Fig. 5). Under these conditions, the viability of cells was not significantly affected (data not shown). Therefore, RCE seems to modulate not only the differentiation of osteoblasts, but also of osteoclasts, to maintain bone balance.

Estrogen plays a fundamental role in the growth and maturation of bone as well as in the regulation of bone turnover in adult bone. Estrogen deficiency is associated with a chronic inflammatory status by increasing the local production of various cytokines, free radicals, and growth factors (Kelly, 1996; Riggs et al., 2002). Proinflammatory cytokines are one critical group of proteins that contribute to the osteoclastogenesis in states of estrogen deficiency. It was reported that estrogen deficiency leads to an up-regulation of IL-1, IL-6, M-CSF, 

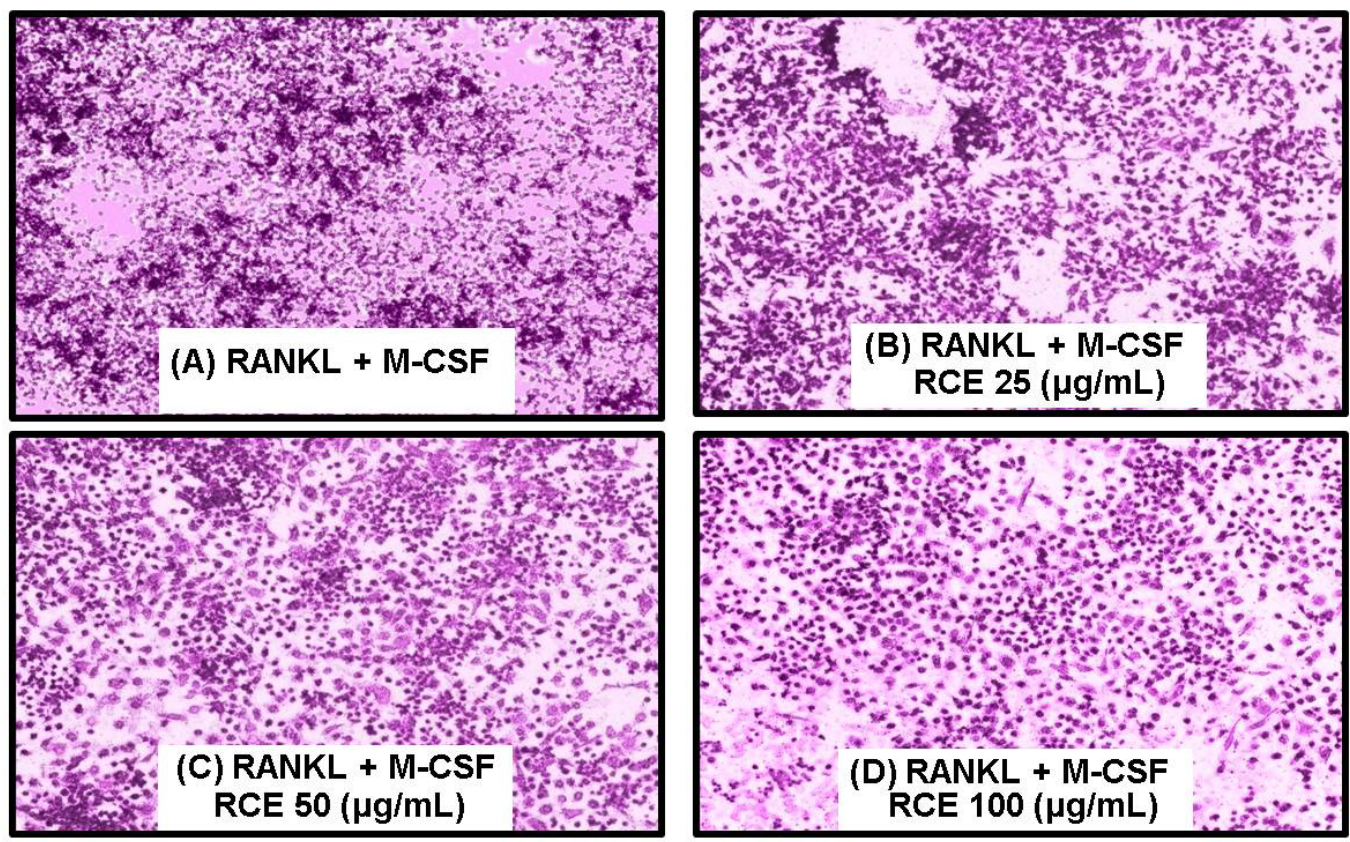

Fig. 5. Effect of RCE on M-CSF/RANKL-induced differentiation of pre-osteoclast RAW 264.7 cells. RAW 264.7 cells were cultured in 24-well dishes at a density of $1 \times 10^{4}$ cells per well and allowed to adhere overnight. To induce osteoclastic differentiation, RAW 264.7 was cultured with M-CSF/RANKL. After 2 days in M-CSF/RANKL media, RCE was added to the cultures and after 4 days in culture, TRAP staining was performed. TRAP-positive multinucleated osteoclasts was detected by microscopy.
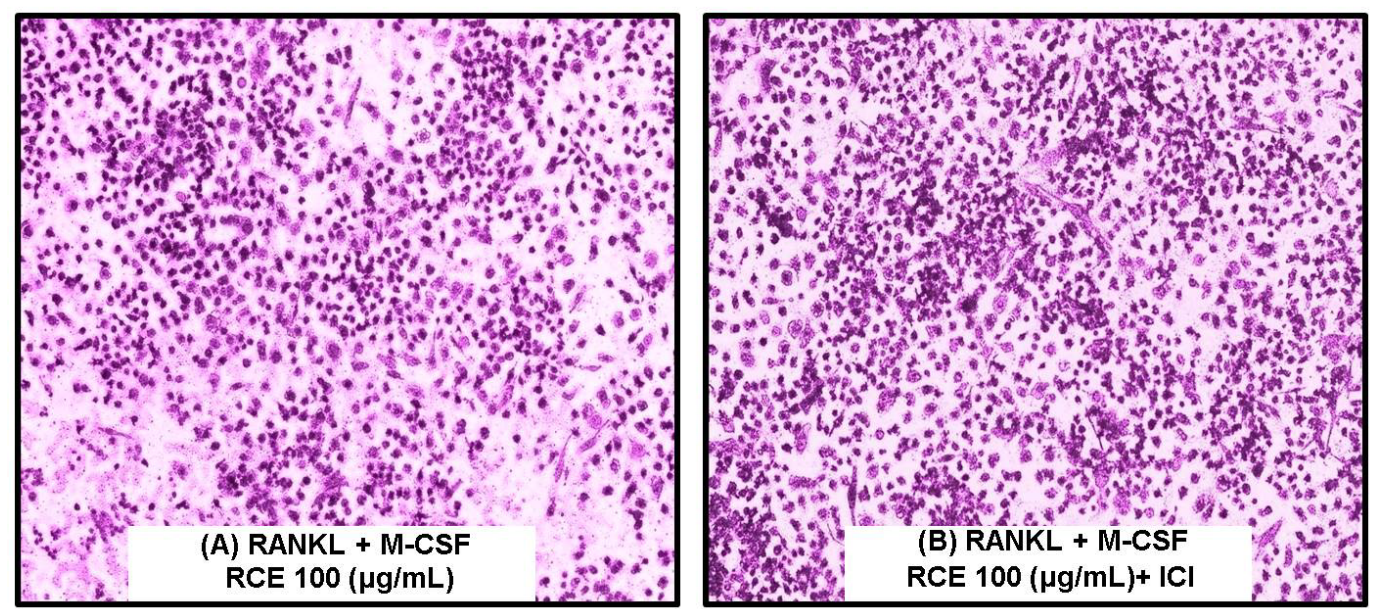

Fig. 6. Effects of RCE on the differentiation of osteoclast involved the estrogen receptor (ER)-a. RAW 264.7 cells were cultured in 24-well dishes at a density of $1 \times 10^{4}$ cells per well and allowed to adhere overnight. To induce osteoclastic differentiation, RAW 264.7 was cultured with M-CSF/RANKL. After 2 days in M-CSF/RANKL media, RCE and ICI were added to the cultures and after 4 days in culture, TRAP staining was performed. TRAP-positive multinucleated osteoclasts was detected by microscopy.

and TNF-a which enhances bone resorption in preosteoclasts. In particular, TNF-a up-regulates stromal cell productions of RANKL and M-CSF, and augments the responsiveness of osteoclast precursors to RANKL (Hotokezaka et al., 2007).

In order to investigate whether the inhibitory effects of
RCE on the differentiation of osteoclast directly related to the estrogen receptor (ER)-a, we performed M-CSF/RANKLinduced formation of osteoclast-like multinuclear cells from RAW 264.7 cells in the presence of selective ER- $a$ antagonist ICI 182,780 (ICI). Various lines of evidence demonstrated 
that ICI caused a robust degradation of ER-a (Kansra et al., 2005; Kansra et al., 2010). In particular, Oliveira et al. (2003) has shown that ICI caused a gradual but dramatic decrease in ER-a expression, but no change in ER-ß. As shown in Fig 6, the inhibitory effect of RCE $(100 \mu \mathrm{g} / \mathrm{ml})$ was reduced by ICI $(10 \mathrm{nM})$ treatment. This suggested that ER- $\alpha$ is partially associated with the inhibitory effect of RCE on the osteoclast differentiation.

Our results suggested that unripe fruits of $R$. coreanus may act beneficial effects on bone mass by regulating both osteoblast and osteoclast. Moreover, it could be promising candidates as a potential preventive agent for post-menopausal osteoporosis.

\section{References}

Aubin, J.E. and J.T. Triffitt. 2002. Mesenchymal stem cells and osteoblast differentiation: In Bilezikian, J.P., L.G. Raisz and G.A. Rodan (eds.), Principles of Bone Biology, 2nd edn. Academic Press, San Diego, USA. pp. 59-81.

Choi, C., H. Lee, H. Lim, S. Park, J. Lee and S. Do. 2012. Effect of Rubus coreanus extracts on diabetic osteoporosis by simultaneous regulation of osteoblasts and osteoclasts. Menopause 19:1043-1051.

Do, S.H., J.W. Lee, W.I. Jeong, J.Y. Chung, S.J. Park, I.H. Hong, S.K. Jeon, I.S. Lee and K.S. Jeong. 2008. Boneprotecting effect of Rubus coreanus by dual regulation of osteoblasts and osteoclasts. Menopause 15:676-683.

Fraser, L.A., K.N. Vogt, J.D. Adachi and L. Thabane. 2011. Fracture risk associated with continuation versus discontinuation of bisphosphonates after 5 years of therapy in patients with primary osteoporosis: a systematic review and metaanalysis. Ther. Clin. Risk Manag. 7:157-166.

Geller, S.E. and F. Kronenberg. 2003. Toward optimal health: the experts discuss the use of botanicals by women. J. Womens Health (Larchmt) 12:847-852.

Hamdy, N.A. 2007. Targeting the RANK/RANKL/OPG signaling pathway: a novel approach in the management of osteoporosis. Curr. Opin. Investig. Drugs 8:299-303.

Horwood, N.J., J. Elliott, T.J. Martin and M.T. Gillespie. 1998. Osteotropic agents regulate the expression of osteoclast differentiation factor and osteoprotegerin in osteoblastic stromal cells. Endocrinology 139:4743-4746.

Hotokezaka, H., E. Sakai, N. Ohara, Y. Hotokezaka, C. Gonzales,
K. Matsuo, Y. Fujimura, N. Yoshida and K. Nakayama. 2007. Molecular analysis of RANKL-independent cell fusion of osteoclast-like cells induced by TNF-alpha, lipopolysaccharide, or peptidoglycan. J. Cell. Biochem. 101: 122-134.

Jang, J.H., J.H. Kim, B.H. Ahn and J.S. Lee. 2011. Physiological functionalities of Vitis hybrid (Sheridan)-Rubus coreanus red wine made by Saccharomyces cerevisiae. Mycobiology 39:109-112.

Jung, K.A., D. Han, E.K. Kwon, C.H. Lee and Y.E. Kim. 2007. Antifatigue effect of Rubus coreanus Miquel extract in mice. J. Med. Food 10:689-693.

Kansra, S., S. Yamagata, L. Sneade, L. Foster and N. Ben Jonathan. 2005. Differential effects of estrogen receptor antagonists on pituitary lactotroph proliferation and prolactin release. Mol. Cell. Endocrinol. 239:27-36.

Kansra, S., S. Chen, M.L. Bangaru, L. Sneade, J.A. Dunckley and N. Ben-Jonathan. 2010. Selective estrogen receptor down-regulator and selective estrogen receptor modulators differentially regulate lactotroph proliferation. PLoS One 5:e10060

Kelly, P.J. 1996. Is osteoporosis a genetically determined disease? Br. J. Obstet. Gynaecol. 103:20-26.

Kim, S., C.K. Kim, K.S. Lee, J.H. Kim, H. Hwang, D. Jeoung, J. Choe, M.H. Won, H. Lee, K.S. Ha, Y.G. Kwon and Y.M. Kim. 2013. Aqueous extract of unripe Rubus coreanus fruit attenuates atherosclerosis by improving blood lipid profile and inhibiting NF- $\kappa$ B activation via phase II gene expression. J. Ethnopharmacol. 146:515-524.

Karsenty, G. 2003. The complexities of skeletal biology. Nature 423:316-318.

Kearns, A.E., S. Khosla and P.J. Kostenuik. 2008. Receptor activator of nuclear factor kappa $B$ ligand and osteoprotegerin regulation of bone remodeling in health and disease. Endocr. Rev. 29:155-192.

Khosla, S. 2001. Minireview: the OPG/RANKL/RANK system. Endocrinology 142:5050-5055.

Kostenuik, P.J. and V. Shalhoub. 2001. Osteoprotegerin: a physiological and pharmacological inhibitor of bone resorption. Curr. Pharm. Des. 7:613-635.

Koo, H.J., E.H. Sohn, Y.J. Kim, S.A. Jang, S. Namkoong and S.C. Kang. 2014a. Effect of the combinatory mixture of Rubus coreanus Miquel and Astragalus membranaceus Bunge extracts on ovariectomy-induced osteoporosis in mice and anti-RANK signaling effect. J. Ethnopharmacol. 151:951-959. 
Koo, H.J., S.C. Kang and E.H. Sohn. 2013. The optimal combination of the mixture of unripe Rubus coreanus and Astragalus membranaceus in the activation and differentiation of osteoblastic cells. Korean J. Plant Res. 26:658-662 (in Korean).

Koo, H.J., S.C. Kang, S.A. Jang, J.E. Kwon, E. Sohn and E.H. Sohn. 2014b. Effects of protocatechuic acid derived from Rubus coreanus on the lipid metabolism in high cholesterol diet-induced mice. Korean J. Plant Res. 27:271-278.

Lee, J.E., S.M. Cho, E. Park, S.M. Lee, Y. Kim, J.H. Auh, H.K. Choi, S. Lim, S.C. Lee and J.H. Kim. 2014. Anti-inflammatory effects of Rubus coreanus Miquel through inhibition of NF$\kappa \mathrm{B}$ and MAP Kinase. Nutr. Res. Pract. 8:501-508.

Lee, K.H. and E.M. Choi. 2006. Rubus coreanus Miq. extract promotes osteoblast differentiation and inhibits bone-resorbing mediators in MC3T3-E1 cells. Am. J. Chin. Med. 34:643-654.

Marsden, J. 2002. The menopause, hormone replacement therapy and breast cancer. J. Steroid Biochem. Mol. Biol. 83:123-132.

McNamara, L.M. 2010. Perspective on post-menopausal osteoporosis: establishing an interdisciplinary understanding of the sequence of events from the molecular level to whole bone fractures. J. R. Soc. Interface 7:353-372.

Miksicek, R.J. 1994. Interaction of naturally occurring nonsteroidal estrogens with expressed recombinant human estrogen receptor. J. Steroid Biochem. Mol. Biol. 49:153-160.

Oliveira, C.A., R. Nie, K. Carnes, L.R. Franca, G.S. Prins, P.T. Saunders and R.A. Hess. 2003. The antiestrogen ICI 182,780 decreases the expression of estrogenreceptor-alpha but has no effect on estrogenreceptor-beta and androgen receptor in rat efferent ductules. Reprod. Biol. Endocrinol. 1:75.

Park, J.H., S.M. Oh, S.S. Lim, Y.S. Lee, H.K. Shin, Y.S. Oh
N.H. Choe, J.H. Park and J.K. Kim. 2006. Induction of heme oxygenase-1 mediates the anti-inflammatory effects of the ethanol extract of Rubus coreanus in murine macrophages. Biochem. Biophys. Res. Commun. 351:146-152.

Riggs, B.L., S. Khosla and L.J. Melton III. 2002. Sex steroids and the construction and conservation of the adult skeleton. Endocr. Rev. 23:279-302.

Simonet, W.S., D.L. Lacey, C.R. Dunstan, M. Kelley, M.S. Chang, R. Lüthy, H.Q. Nguyen, S. Wooden, L. Bennett, T. Boone, G. Shimamoto, M. DeRose, R. Elliott, A. Colombero, H.L. Tan, G. Trail, J. Sullivan, E. Davy, N. Bucay, L. RenshawGegg, T.M. Hughes, D. Hill, W. Pattison, P. Campbell, S. Sander, G. Van, J. Tarpley, P. Derby, R. Lee and W.J. Boyle. 1997. Osteoprotegerin: a novel secreted protein involved in the regulation of bone density. Cell 89:309-319.

Thomas, G.P., S.U. Baker, J.A. Eisman and E.M. Gardiner. 2001. Changing RANKL/OPG mRNA expression in differentiating murine primary osteoblasts. J. Endocrinol. 170:451-460.

Udagawa, N., N. Takahashi, H. Yasuda, A. Mizuno, K. Itoh, Y. Ueno, T. Shinki, M.T. Gillespie, T.J. Martin, K. Higashio and T. Suda. 2000. Osteoprotegerin produced by osteoblasts is an important regulator in osteoclast development and function. Endocrinology 141:3478-3484.

Wensel, T.M., M.M. Iranikhah and T.W. Wilborn. 2011. Effects of denosumab on bone mineral density and bone turnover in postmenopausal women. Pharmacotherapy 31: 510-523.

Yang, H.M., S.M. Oh, S.S. Lim, H.K. Shin, Y.S. Oh and J.K. Kim. 2008. Antiinflammatory activities of Rubus coreanus depend on the degree of fruit ripening. Phytother. Res. 22:102-107. 